\title{
Growth and Characterization of Urea L-Valine An Organic non Linear Optical Crystal
}

\author{
R.N. JAYAPRAKASH ${ }^{1 *}$ and P. KUMARADASS ${ }^{2}$ \\ 1'Department of Physics,Adhiyamaan College of Engineering,Hosur - 6351 09, India. \\ ${ }^{2}$ Department of Physics, Periyar University, Salem, India. \\ *Corresponding author: prakashnandhi@yahoo.co.in \\ http://dx.doi.org/10.13005/ojc/290416
}

(Received: August 30, 2013; Accepted: October 15, 2013)

\begin{abstract}
Single crystals of new organic nonlinear optical material urea L-valine were grown by slow eveporation solution growth technique at room temperature.Elemental compostion percentages were analysed using $\mathrm{CHN}$ study. Crystalline nature of the sample was confirmed by powder XRD. A functional group present in the crystal was identified using FT-IR spectral analysis. An optical transmittance study was also carried out for the crystal. Dielectric studies were performed with respect to temperature at various frequencies $(50 \mathrm{~Hz}-5 \mathrm{MHz})$. Photoconductivity study reveals the negative photoconductivity nature of the crystal. Powder second generation efficiency of the crystal was studied using modified kurtz and perry technique.
\end{abstract}

Key words: Nonlinear, Powder X-ray diffraction, Optical, Dielectric, Photoconductivity, Single crystal XRD.

\section{INTRODUCTION}

The search for new organic nonlinear optical crystals has been of great interest because of their wide range of potential applications such as frequency doubling, optical switching, optical disk data storage, optical modulation, laser remote sensing and medical diagnostics. These materials attracting significant attention as they posses large nonlinear optical susceptibilities, ultrafast nonlinear response time, high laser damage threshold and scope for introducing desirable characteristics by multifunctional substitutions ${ }^{1-3}$. For NLO device applications still there is a demand for good quality crystals with higher efficiency. For the past ten years amino acids are playing a vital role in the nonlinear optical crystal growth. They individually exhibit nonlinear properties as they posses proton donor carboxyl acid (COO) and proton acceptor amino $\left(\mathrm{NH}_{3}\right)$ group also in solid state they exist as zwitterions. Amino acid crystals play a major role in NLO applications such as L-valine, L-alanine, Lproline, L-leucine, L-histidine, L-arginine and L-phenylalanine. Worldwide urea and its derivatives are extensively used in crystal engineering and supramolecular chemistry for their flexibility in the synthesis of functional materials. Also for a long time it is used as a model system for crystals from solution growth and reference material in Powder SHG ${ }^{3-9}$. 
In the present work urea and L-valine single crystals were grown by slow evaporation solution growth technique. The grown crystal was characterized by various characterization techniques such as CHN analysis, powder XRD, FT-IR, optical transmittance, dielectric, photoconductivity and powder SHG measurements.

\section{MATERIALS AND METHODS}

\section{Crystal growth}

Urea L-valine single crystals were grown by slow evaporation solution growth technique at room temperature. Urea and L-valine were seperately taken in equilmolar ratio and dissolved in double distilled water and stirred seperately for half an hour. Then the solutions were mixed together and stirred well for about $5 \mathrm{hrs}$. the saturated solution was filtered using whatman (No:42 grade) filter paper. The filtered solution was kept at undisturbed place for crystal growth. After two weeks optically good qulity single crystals were obtained from the mother solution. Fig. 1 shows the quality of the crystal.

\section{RESULT AND DISCUSSION}

\section{CHN analysis}

The elemental composition percentage of urea L-valine crystals were analysed using Vario $E L$ III Elemental analyser. The experimental C, H and N percentages were compared with theoritical values and listed in Table 1. Which confirms the prescence of the compound.

\section{Powder XRD}

Powder X-ray diffraction studies for the grown crystals were carried out using RICH SIEFERT $\mathrm{X}$-ray powder diffractometer using CuK \pm (»=1.5405 $\AA$ ) radiation. Sample was scanned over 2 , the range of $5-80$ at rate of $3 æ \% / m i n$. The crystals were finely grained into powder in uniformed size and subjected to analysis. Crystalline nature was quite clear from the occurrence of sharp peaks at specific Bragg's angles. Fig. 2 shows the recorded spectrum. The powder XRD result of this crystal was compared with previous reports in which urea and L-valine were mixed with acids and metals. From the XRD peak intensity it is concluded that in the present work crystalline nature was very high while comparing previous reports ${ }^{10-15}$.

\section{FT-IR analysis}

The middle IR spectrum of urea L-valine crystal is carried out at room temperature in the spectral range of $4000-400 \mathrm{~cm}^{-1}$ by $\mathrm{KBr}$ pellet technique. The recorded spectrum is shown in Fig. 3 . The band observed at 3344 and $3444 \mathrm{~cm}^{-1}$ are assigned to $\mathrm{OH}$ and $\mathrm{NH}_{2}$ symmetric and asymmetric stretching vibrations. The torsional mode of $\mathrm{NH}_{3}$ is observed at $543 \mathrm{~cm}^{-1}$. The peak at $669 \mathrm{~cm}^{-1}$ is due to $\mathrm{OH}$ bending vibration. The symmetric and asymmetric stretching vibrations of $\mathrm{CH}_{2}$ are observed at 2881 and $2947 \mathrm{~cm}^{-1}$. Bands observed at 1029 and $1469 \mathrm{~cm}^{-1}$ are assigned to $\mathrm{N}-\mathrm{C}-\mathrm{N}$ symmetric and asymmetric stretching vibration of urea. Frequencies at 1431 and $1327 \mathrm{~cm}^{-1}$ are assigned to $\mathrm{C}-\mathrm{O}$ stretching vibration of $\mathrm{L}$-valine. The symmetric and asymmetric stretching of COO-vibrations (carboxylate anion) are found at 1585 and $1431 \mathrm{~cm}^{-1}$ respectively. The absorption band at 1068 and $1585 \mathrm{~cm}^{-1}$ are assigned to $\mathrm{CN}$ and $\mathrm{C}=\mathrm{O}$ stretching vibrations respectively. $\mathrm{CH}_{2}$ bending vibration is observed at $1350 \mathrm{~cm}^{-1}$. $\mathrm{NH}_{2}$ in-plane bending vibration appears at $1585 \mathrm{~cm}^{-1}$. The C-C skeletal vibration is observed at $775 \mathrm{~cm}^{-1}$. The peak around $1145 \mathrm{~cm}^{-1}$ is due to $\mathrm{NH}_{2}$ rocking vibration. COO- rocking vibration could be assigned to peaks 543 and $428 \mathrm{~cm}^{-1}$. The frequencies at 713 , 821 and $948 \mathrm{~cm}^{-1}$ are correspond to $\mathrm{CH}$ out-of-plane bending vibration.

\section{Optical studies}

Optical transmittance is one of the important desirable properties for identifying the potential NLO material. Optical transmittance studies were carried out in the wavelength range between 200-1100nm using a Perkin-Elmer lambda 35 spectrometer. Fig. 4 represents the transmittance spectrum of urea L-valine crystal. Crystal exhibits large transmittance in the visible region, possessing a transmittance greater than $80 \%$ for incident wavelength ranging from 300 to $1200 \mathrm{~nm}$. Lower cut off wavelength of

Table1: Elemental composition percentage of urea L-valine crystals

\begin{tabular}{lcc}
\hline Elements & Experimental(\%) & Theoretical (\%) \\
\hline Carbon & 40.78 & 40.67 \\
Hydrogen & 8.42 & 8.53 \\
Nitrogen & 23.6 & 23.71 \\
\hline
\end{tabular}


the crystal is $245 \mathrm{~nm}$. Optical band gap $\left(\mathrm{E}_{\mathrm{g}}\right)$ of the material was evaluated by the extrapolation of the linear part ${ }^{16-17}$. Which is represented in Fig. 5 from the graph band gap energy is calculated as $3.03 \mathrm{eV}$. Large transmittance and high band gap is one of the important for materials possessing greater possibilities for nonlinear device applications ${ }^{18-20}$.

\section{Dielectric studies}

Dielectric studies for the crystals were performed using Hioki 3532-50 LCR meter. Measurements were carried out in the frequency range from $50 \mathrm{~Hz}$ to $5 \mathrm{MHz}$ at various temperature (303 to $378 \mathrm{~K}$ ). Dielectric constant of the crystals were calculated using the capacitance value by the given relation

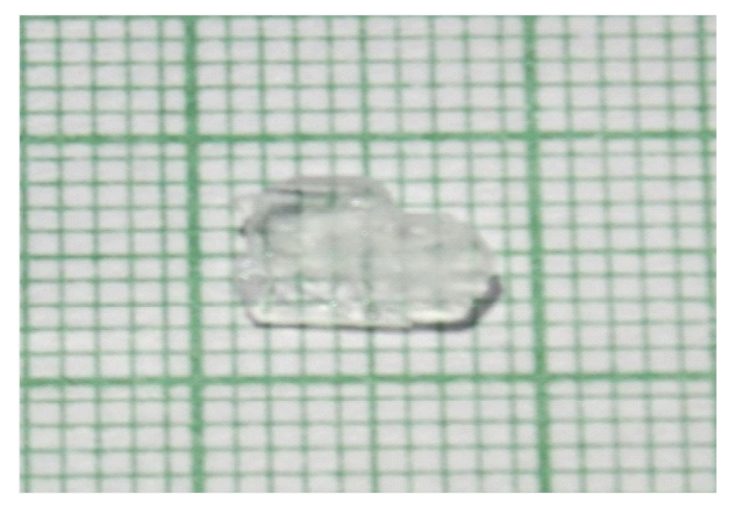

Fig. 1: Photograph of as grown crystal

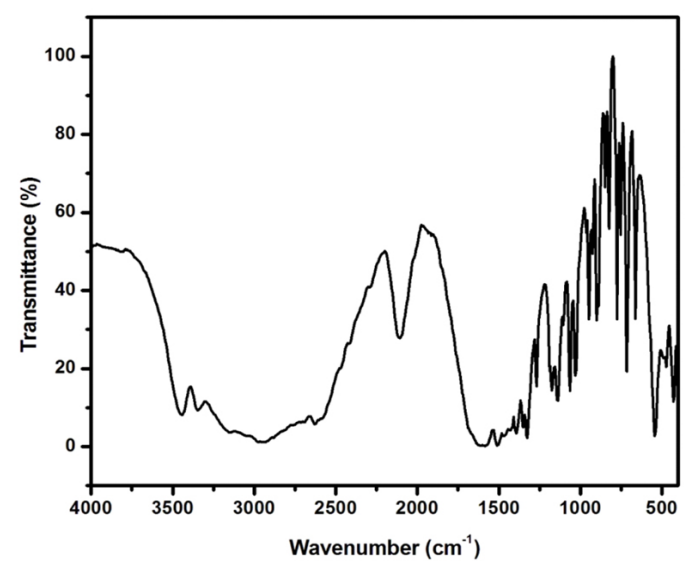

Fig. 3: FT-IR spectrum.
Where $\mathrm{C}$ is the capacitance and $\mathrm{d}$ is the thickness of the sample, $A$ is the cross sectional area of the sample and is the free space permitivity of the sample. Fig. 6 shows dielectric constant with respect to log frequency. From the figure it is observed that dielectric constant is very high at low frequency and becomes low at high frequency (Upto $1 \mathrm{MHz}$ ) and attains minimum value at higher frequencies (Above $1 \mathrm{MHz})$. High dielectric constant at low frequency is due to the prescence of all four polarizations namely electronic, ionic, space charge and orientation. Low value at higher frequency is due to significant loss of these polarizations ${ }^{21}$. Fig. 7 shows the dielectric loss Vs log frequency. The dielectric loss is very low at high frequency it confirms that grown crystal possess less defects with enhanced optical quality. Materials

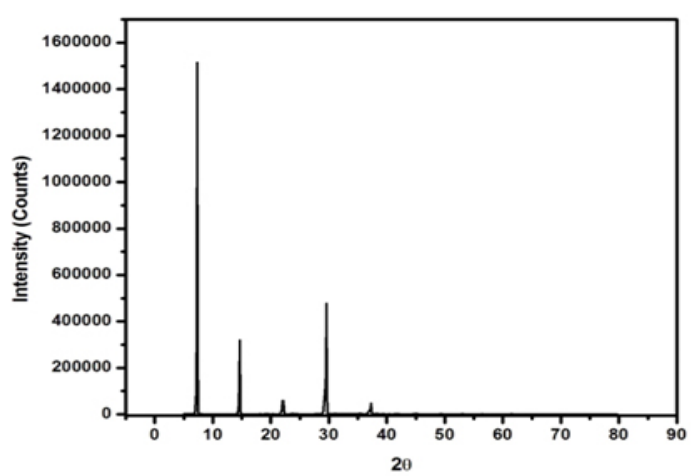

Fig. 2: Powder XRD spectrum

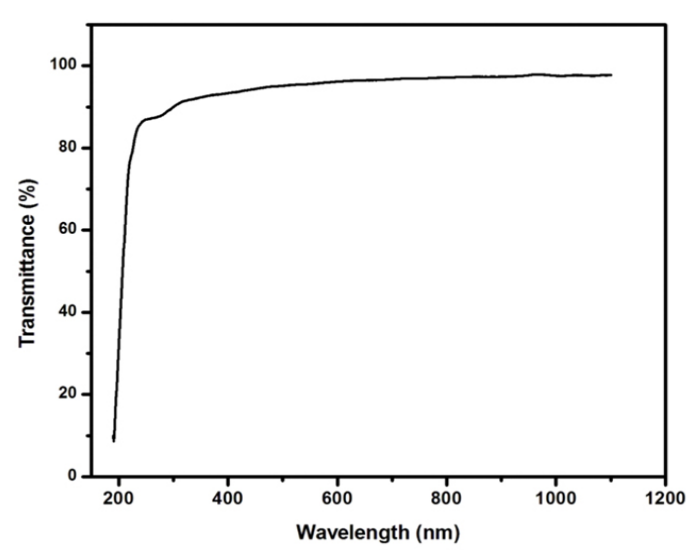

Fig. 4: Optical transmission spectrum 
with high dielectric constant and low dielectric loss can be very much useful for capacitor and heating devices applications ${ }^{22}$.

\section{Photoconductivity}

Photoconductivity studies were carried out at room temperature using Keithley 6512 electrometer. Crystal was silver pasted on opposite faces and thin copper wire was connected both sides on the sample and it is connected in series with electrometer. The sample was protected from all the radiation. The applied input voltage was increased from 10 to $100 \mathrm{~V}$ and resultant dark current was recorded. Then the samples were exposed to radiation from 100W halogen lamp containing iodine vapour and tungston filament. Phtocurrent was recorded for the

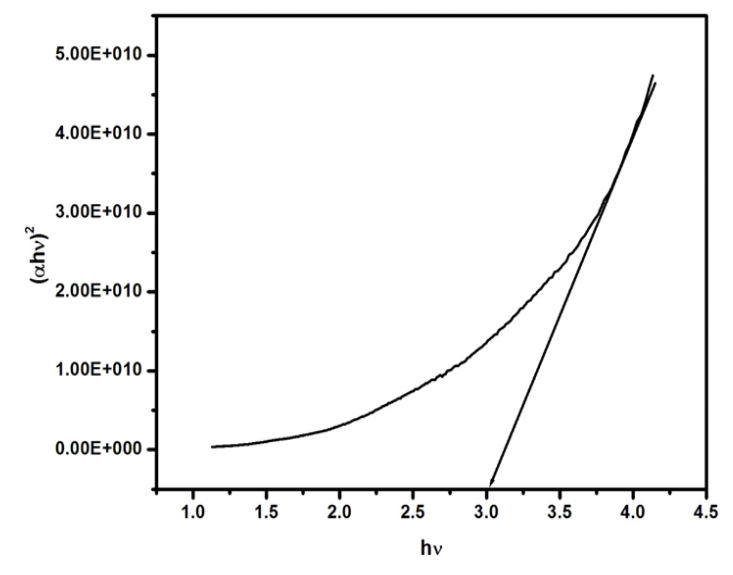

Fig. 5: Determination of optical band gap

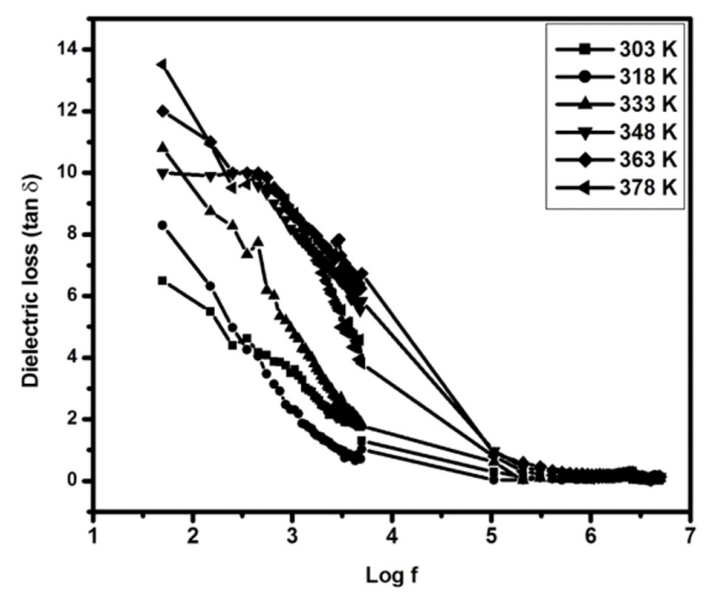

Fig. 7: Dielectric loss Vs Log $f$ same input voltage. Fig. 8 shows the photo current and dark current response for the sample. From the figure it is concluded that dark current is higher than photocurrent and this phenomena is called negative photoconductivity. It may be due to the reduction of charge carriers in the prescence of radiation. Further it was explained by Stockman model ${ }^{23}$. Negative photoconductivity materials can be used for UV and IR detector applications ${ }^{24}$.

\section{Powder SHG}

Powder second harmonic generation efficiency of the grown crystal was determined using modified Kurtz and Perry method ${ }^{25}$. Q-switched mode locked Nd:YAG laser was used as a optical source. The crystals were grained into powder and densly

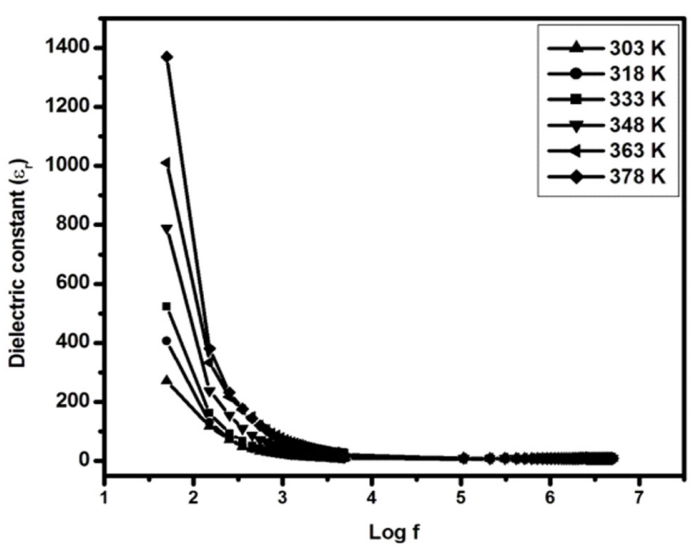

Fig. 6. Dielectric constant Vs Log $f$

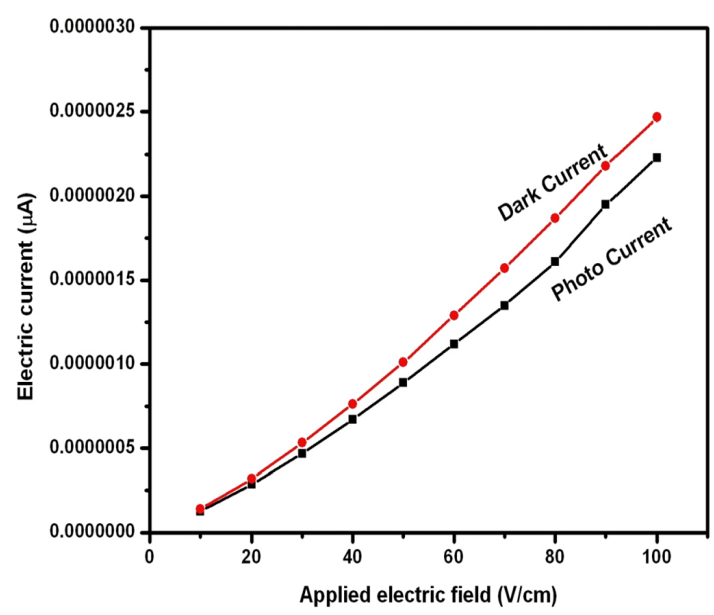

Fig. 8: Photoconductivity response for the 
packed between two glass slides. The input energy was measured using power meter and it is $4.6 \mathrm{~mJ} / \mathrm{s}$. Powdered KDP crystal was used as a reference material. The laser light $(1064 \mathrm{~nm})$ was focussed on to the sample. The green output $(532 \mathrm{~nm})$ was collected by a photomultiplier tube and finally measured on the storage oscilloscope (CRO) as output voltage. Output signal of $15 \mathrm{mV}$ is obtained for the titled compound while the KDP is $7.4 \mathrm{mV}$. It is concluded that title compound is times 2.02 times greater than that of reference KDP.

\section{Single crystal XRD analysis}

The unit cell parameters are determined by using the singlecrystal X-ray diffraction data obtained with a four-circle Nonius CAD4/diffractometer ( MoK $\alpha, \lambda=0.710738 \AA$ ). The unit cell parameters are $a=5.771(1) \AA, b=6.012(1) \AA, c=12.295(5)$ $\AA, \alpha=90^{\circ} \beta=90^{\circ} \gamma=90^{\circ}$ and volume $=426.8(2) \AA^{3}$. From the data, it is observed that the grown crystal is belongs to orthorhombic system.

\section{CONCLUSION}

Urea L-valine single crystals were grown by slow evaporation solution growth technique at room temperature. Crystalline nature of the compound was confirmed by powder XRD analysis. A functional group present in the crystal was identified using FT-IR studies. Optical transmision study confirms the quality of the crystals for NLO applications. Optical band was found to be $3.03 \mathrm{eV}$. In dielectrics dielectric constant is very high at low frequency. Phtoconductivity study reveals the negative photoconductivity nature of the crystal. powder SHG efficiency of the grown crystal is 2.02 times greater than KDP.

\section{ACKNOWLEDGEMENTS}

The authors would like to acknowledge Dr P.K. Das, Indian Institute of Science for NLO studies.

\section{REFERENCES}

1. P. Kumaresan, S. Moorthy Babu, P.M. Anbarasan, Optical Materials 30: 1361-1368 (2008).

2. A. Joseph Arul Pragasam, J. Madhavan, , M. Gulam Mohamed, S. Selvakumar, K. Ambujam, P. Sagayaraj, Optical Materials 29: 173-179 (2006).

3. G. Madhurambal and P. Anbu Srivinasan, Crystal Research Technology 41: 231-235 (2006).

4. S. Pandiarajan, M. Umadevi, R.K. Rajaram, V. Ramakrishnan, Spectrochimica Acta Part A: 5.Molecular and Biomolecular Spectroscopy 62: 630-636 (2005).

6. Sweta Moitra, Tanusree Kar, Journal of Crystal Growth 310: 4539-4543 (2008).

7. G. Bhagavannarayana, B. Riscob, Mohd. Shakir, Materials Chemistry and Physics 126: 20-23 (2011).

8. J. Chandrasekaran, P. Ilayabarathi, P. Maadeswaran, Optoelectronics and Advanced Materials - Rapid Communications 5 (2011) 1325 - 1327.
9. Hiromu Yoshiura, Hiroshi Nagano, Izumi Hirasawa, Journal of Crystal Growth 363: 55-60 (2013).

10. A. Cyrac Peter, M. Vimalan, P. Sagayaraj, J. Madhavan, Physica B 405: 65-71 (2010).

11. J. Chandrasekaran, P. Ilayabarathi, P. Maadeswaran, P. Mohamed Kutty, S. Pari, Optik 123 (2012) 1407-1409.

12. P. Maadeswaran, J. Chandrasekaran, Optik 122: 1128-1131 (2011).

13. A. Puhal Raj, C. Ramachandra Raja, Spectrochimica Acta Part A: Molecular and Biomolecular Spectroscopy 97: 83-87 (2012).

14. Bal Krishan, Om Prakash and E.H.EIMassalamy, Orient J. Chem., 29(1): 381-388 (2013).

15. Om Prakash, S.A. Iqbal G. Jacob, Orient J. Chem., 29(3): 1079-1084 (2013).

16. V.K. Dixit, S. Vanishri, H.L. Bhat, E. de Matos Gomes, M. Belsley, Santinha, G. Arunmozhi, V. Venkataramanan, F. Proena, A. Criado, Journal of Crystal Growth 253: 460-466 
(2003).

17. J.Tauc, Amorphous and Liquid semiconductors, J. Tauc Edition, Plenum, New York, (1974).

18. J. Chandrasekaran, S. Balaprabhakaran, B. Babu, Optik (2013) Article in press.

19. J. Chandrasekarana, B. Babu, S. Balaprabhakaran, P. Ilayabarathi, P.Maadeswaran, K. Sathishkumar, Optik (2012) Article in press.

20. S. Dhanuskodi and K. Vasantha, Crystal research Technology 39: 259-265 (2004).

21. G. Shanmugam, S. Brahadeeswaran, Spectrochimica Acta Part A: Molecular and
Biomolecular Spectroscopy 95: 177-183 (2012).

22. V. Chithambaram, S. Jerome Das, S. Krishnan, Journal of Alloys and Compounds 509: 45434546 (2011).

23. J. Chandrasekaran, S. Balaprabhakaran, B. Babu, Optik (2013) Article in press.

24. V.N. Joshi, Photoconductivity, Marcel Dekker, New York, 1990.

25. B. Babu, J. Chandrasekaran, S. Balaprabhakaran and P. Ilayabarathi, Material Science-Poland 31: 151-157 (2013). 